\title{
How to support the basic sciences
}

\begin{abstract}
It has become axiomatic that basic science faculty and research programs at medical schools must support themselves. The days when excess clinical revenue was used to support basic research are long gone. With the pressure of managed care and faculty practice programs on medical school budgets, it appears that medical school leaders are looking everywhere but at clinical programs for critical support of biomedical research at their institutions, even as NIH support shrinks.
\end{abstract}

The future of both basic science programs and clinical programs at academic medical centers rests in reestablishing a healthy flow of financial support from clinical programs to basic programs. In order for this support to be meaningful and acceptable to clinicians, the relationship between basic and clinical programs has to become a two-way street. For too long, basic scientists have ignored their clinical colleagues while bemoaning the apparent flow of money to clinical programs, and as a result, many basic science programs have limped along as poor cousins.

The time has come for basic scientists at medical centers to provide academic programs for their clinical colleagues, including training in basic research for medical students, house staff, and faculty. Courses highlighting the latest advances in basic research that are relevant to the current practice of medicine need to be available and accessible. Clinicians practicing at academic health centers could benefit from learning about new scientific discoveries, and they would likely recognize that this enriches their profession and enhances their abilities to provide cutting-edge care for patients. Providing such education is both an obligation and an opportunity for basic science faculty; it could enhance their productivity and the relevance of their own work, and it would provide a reason for clinicians to share proceeds from their clinical practices with their basic science colleagues.

The leaders of academic health centers have avoided the uncomfortable truth that within their institutions an MD is likely to make a substantially larger income than a $\mathrm{PhD}$ with equivalent training.
When they are enlisting the support of potential donors, however, it is often the accomplishments of basic scientists that are cited. Much of the prestige garnered by an academic health center rests on the achievements of its basic scientists. Clinicians working at leading academic health centers must come to appreciate that the accomplishments of their basic science colleagues, whose discoveries lead to frontpage stories about medical breakthroughs, provide both tangible and intangible benefits to their practices. Basic scientists need to do a much better job of sharing their expertise and imparting the excitement of discovery to their clinical colleagues. Bowing under the heavy load of their clinical practices, many clinicians may have had to abandon the dreams they had in medical school of finding new cures for disease, yet the sense of curiosity and the desire to learn is never lost. To the extent that welldesigned, timely, and efficient programs offered by basic scientists can involve clinicians in the quest for knowledge, the entire health care enterprise could benefit.

Improved relationships between basic and clinical faculty could also enable the leaders of academic health centers to develop a more focused approach to fundraising for basic science programs. Currently, grateful patients are most likely to make substantial donations to health centers through their doctors. Unfortunately, if these clinicians are not connected to any meaningful research programs, the precious resources may end up paying for cosmetic enhancements of the facility rather than supporting programs that could uncover a new treatment for an important disease and truly fulfill the dreams of the donor. By working together, clinicians and basic scientists, with guidance from the central administration, could strengthen the impact of philanthropic support on academic health centers by targeting it to the most significant research programs.

In order for such a program to work, clinicians and basic scientists need to foster a relationship of mutual understanding and respect. Basic scientists can take the first step, for they can translate what they do in a way that can be of immediate use and interest to clinicians. The next step requires leadership from the deans at medical schools to create institutional collaborations between clinical and basic science departments that allow them to value each other's needs and to share intellectual and financial resources. If the leaders are philosophically committed to supporting this cooperative effort, they can overcome the reluctance to impose new taxes on clinical income, and they will be able to direct philanthropic dollars where they are most needed.

Unifying basic scientists and clinicians on the campuses of academic health centers is an effort that must succeed. If we fail at this task, then the promise of what modern biomedical research coupled with patient care can achieve will not be realized. The public and the policy makers will lose faith in the ability of academic health centers to deliver new cures, and the biomedical research infrastructure will crumble. To win this battle, academic health center leaders will need to push the agenda of collaboration to new levels that require a long-range altruistic perspective. We need to leave the next generation of biomedical researchers a culture in which scientists and physicians walk arm-in-arm down the corridors of our medical schools, exchanging ideas, teaching together, and teaching each other.

\section{Andrew R. Marks \\ Editor-in-Chief}

